The 1993 Nobel Prize in Physics was awarded to R.A. Hulse and J.H. Taylor for the "discovery of a new type of pulsar, a discovery that has opened up new possibilities for the study of gravitation". In subsequent work, general relativity passed new, strong-field tests with complete success.

\title{
A Laboratory for Relativity
}

Almost 20 years ago, Joseph Taylor and Russell Hulse from Princeton University discovered, in a systematic search for new pulsars using a large radiotelescope, the binary radio pulsar PSR $1913+16$ that appears to have been exquisitely designed as a laboratory for general relativity [1]. In subsequent years, much effort has been put into measuring its pulse arrival times with increasing precision and comparing the results with general relativity.

Before entering into a brief description of the analysis and an appreciation of the importance of the results, we shall recall the gross features of the binary pulsar system. The nominal period of the pulsar is $59 \mathrm{msec}$. This short period was observed to be periodically shifted, which proved that the pulsar is a member of a binary system with an orbital period of 7.75 hours (precise numbers for various orbital parameters are given in Table 1). With Kepler's third law and reasonable masses one concludes that the orbit is not much larger than the Sun's diameter.

Correspondingly, the velocity of the pulsar is about $10^{-3} \mathrm{c}$ and it moves through a relatively strong gravitational field (about 100 times stronger than the gravitational field of the Sun near Mercury). These numbers show that several special and general relativistic effects should be observable - a goal that has been explored since 1974 with increasing accuracy. It turned out that PSR $1913+16$ is one partner of a "clean" binary system of two neutron stars each having approximately 1.4 solar masses $M_{\bullet}$ (see Fig. 1). In addition, PSR $1913+16$ behaved very well all the time, for it has not made any discontinuous jumps (glitches) nor generated noise since being discovered.

From the shape of the radial velocity curve which is derived from the periodically changing pulse arrival rate due to the Doppler shift, Hulse and Taylor noticed very soon that the orbit is highly eccentric (an aspect ratio $e \approx 0.62$ ). One of the main reasons why the Hulse-Taylor pulsar has become an ideal testing ground for general relativity is that there are regions of very strong field in the two-body system: at the surface of the neutron star the characteristic measure for the field strength $G \times$ mass $/\left(c^{2} \times\right.$ radius $)$ becomes about 0.2 which is close to the maximum value of 0.5 at the horizon of a black hole, where $G$ is the gravitational constant.

\section{Arrival Time Analysis}

Pulsars represent natural clocks which are remarkably stable. Owing mainly to magnetic dipole radiation, the period of the rotating magnetized neutron star increases

Norbert Straumann has been Professor of Theoretical Physics in the Institute of Theoretical Physics, University of Zurich, Winterthurerstrasse 190, CH-8057 Zurich, since 1969.

\section{N. Straumann}

Institute for Theoretical Physics, University of Zurich

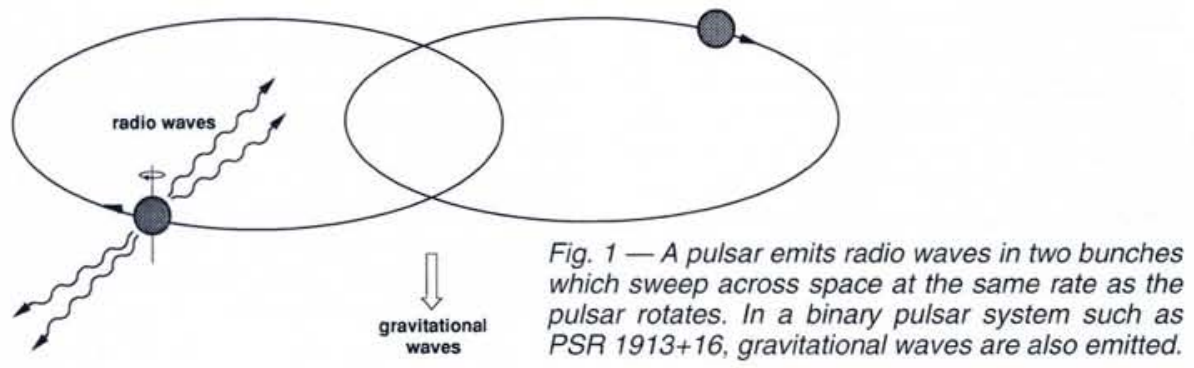

slowly, and hence the intrinsic pulsar frequency $v$ decreases steadily with time. For PSR $1913+16$ the first derivative with time is very small, but measurable: $\dot{v}=-2.47583(2)$ $x 10^{-15} \mathrm{sec}^{-2}$. The pulsar is thus almost as stable as the best atomic clock. The sequence of arrival times on Earth of the electromagnetic pulses emitted by the orbiting pulsar is, however, slightly distorted by a number of effects. Besides the almost periodic Doppler modulation which is influenced by general relativistic effects of the orbital motion, the pulse originating from the vicinity of the pulsar (strong-field region) travels afterwards through the relatively weak-field region between the condensed objects, and finally through the weak field of the solar system. The arrival time $\tau_{N}^{\text {Earth }}$ of the $N$ th pulse on Earth depends therefore on many parameters $p_{i}$, which include a detailed description of the orbit of the binary system

$$
\tau_{N}^{\text {Earth }}=F\left(N ; p_{1}, p_{2}, \ldots\right) .
$$

In order to obtain an explicit timing formula, one must first transform each observatory-centred time of arrival $\tau$ Earth to the reference frame of the solar system barycentre $\tau_{N}^{\text {bary }}$, and then to the pulsar comoving frame. For the latter frame, the time $T_{N}$ of emission of the pulse number $N$ is implicitly parametrized by the first few terms of a Taylor series

$$
N=N_{0}+v T_{N}+\dot{v} T_{N}^{2} / 2+\ddot{v} T_{N}^{3} / 6
$$

The high-quality data obtained by Taylor and coworkers [1-4] forced theoreticians to work out an increasingly accurate timing formula (Eq. 1), which takes into account all the effects mentioned above. Initial derivations [5] were based on everywhere weakfield assumptions, but later the influence of the strong-field region of emission on the final timing formula was also carefully analyzed [6]. The theoretical problems involved are difficult because a very accurate general relativistic description of a two-body system, consisting of compact objects, has to be derived from Einstein's field equations. However, space limitations do not allow a discussion of the theoretical issues involved (see [7] and references therein for a full treatment).

The final form of the timing expression (Eq. 1), whose accuracy matches the experimental state-of-the-art, contains a large number of parameters. Besides less interesting quantities such as $v, \dot{v}$, the dispersion constant describing the frequencydependent delay owing to the interstellar plasma, celestial coordinates, proper motion and annual parallax, there also appear 13 orbital parameters which are - in principle - measurable. Among these, five show up even in a purely Keplerian analysis of the

Table 1 - Measured orbital parameters of two binary pulsar systems. Figures in parentheses represent uncertainties in the last quoted digit; those in square brackets represent expected values of unmeasured quantities, according to general relativity. (From [4].)

\begin{tabular}{lcl}
\hline Parameter & PSR B1534+12 & PSR B1913+16 \\
\hline & Keplerian parameters & \\
Orbital period, $P_{b}(\mathrm{~s})$ & $36351.70270(3)$ & $27906.9807804(6)$ \\
Eccentricity, $e$ & $0.2736779(6)$ & $0.6171308(4)$ \\
Projected semi-major axis, $x(\mathrm{~s})$ & $3.729468(9)$ & $2.3417592(19)$ \\
Time of periastron, $T_{o}(\mathrm{MJD})$ & $48262.8434966(2)$ & $46443.99588319(3)$ \\
Longitude of periastron, $\omega\left(^{\circ}\right)$ & $264.9721(16)$ & $226.57528(6)$ \\
& Post-Keplerian parameters & \\
Advance of periastron, $\dot{\omega}\left({ }^{\circ} \mathrm{yr}^{-1}\right)$ & $1.7560(3)$ & $4.226621(11)$ \\
Time dilation, $\gamma(\mathrm{ms})$ & $2.05(11)$ & $4.295(2)$ \\
Orbital period derivative, $\dot{P}_{b}\left(10^{-12}\right)$ & $-0.1(6)$ & $-2.422(6)$ \\
Range of Shapiro delay, $r(\mu \mathrm{s})$ & $6.2(1.3)$ & {$[6.836]$} \\
Shape of Shapiro delay $s \equiv \sin i$ & $0.986(7)$ & {$[0.734]$} \\
\hline
\end{tabular}


orbital motion and are readily measured with high accuracy (see Table 1). The remaining eight "post-Keplerian" parameters are of great interest for general relativity. In the parametrization used by Damour and Taylor [8], these are the four secular derivatives (the advance of periastron $\dot{\omega}$, the orbital period derivative $P_{\mathrm{b}}$, the derivative $\dot{x}$ of the projected semi-major axis $x$, and $\dot{e})$, the Einstein parameter $\gamma$ describing the combined effect of the gravitational redshift and the transverse Doppler effect, the "range" $r$, the "shape" $s$ of the orbital Shapiro delay, and an orbital shape correction $\delta_{\theta}$.

At this point the following fact has to be stressed: seven quantities are needed to fully specify the dynamics of the two-body system (up to an uninteresting rotation about the line of sight). Therefore, the measurement of any two post-Keplerian parameters, besides the five Keplerian ones, allows the remaining six parameters to be predicted. Thus, if one is able to measure $P$ post-Keplerian parameters one has $P$-2 distinct tests of general relativity (or of any alternative relativistic gravity theory).

\section{Timing Measurements of PSR 1913+16}

Since PSR $1913+16$ is one of the weakest pulsars $(\approx 0.7 \mathrm{mJy}$ at $1400 \mathrm{MHz})$, Taylor and coworkers used for their observations the large $305 \mathrm{~m}$ spherical reflector of the Arecibo Observatory in Puerto Rico. Background noise, propagation effects in the interstellar medium and the steep radiofrequency spectrum limit accurate pulsar timing observations to the one-decade frequency interval $300 \mathrm{MHz}-3 \mathrm{GHz}$.

Individual pulses are weak and have a complicated pulse profile, but averaging over several minutes leads to a relatively regular mean-pulse profile. This is then fitted by the method of least squares to a long-term average "standard profile" to obtain the precise arrival time. All timing measurements since February 1981 have errors not greater than $20 \mu \mathrm{s}$. Because the estimated uncertainties of individual times of arrival are subject to errors, daily average values of uncertainties are taken and then scaled by the known dependence of telescope gain and system noise temperature on the zenith angle. The following brief description of the observation campaigns is taken from a recent paper by Taylor [4]:

"In the timing observations that my colleagues and I make regularly at Arecibo, radio-frequency voltages induced in the feed antennas are amplified, converted to intermediate frequency, and passed

Table 2-Comparison of the observed orbital period derivative of PSR1913+16 with the general relativistic prediction. (From [4].)

\begin{tabular}{ll}
\hline Parameter & $\left(10^{-12}\right)$ \\
\hline Observed value, $\dot{P}_{b}^{\text {obs }}$ & $-2.4225 \pm 0.0056$ \\
Galactic contribution, $\dot{P}_{b}^{\text {gal }}$ & $-0.0124 \pm 0.0064$ \\
Intrinsic orbital period & \\
decay, $\dot{P}_{b}^{\text {obs }}-\dot{P}_{b}^{\text {gal }}$ & $-2.4101 \pm 0.0085$ \\
General relativistic prediction \\
$\dot{P}_{b}^{G R}$ & $-2.4025 \pm 0.0001$ \\
$\left(\dot{P}_{b}^{\text {obs }}-\dot{P}_{b}^{\text {gal }}\right) / \dot{P}_{b}^{G R}$ & $1.0032 \pm 0.0035$ \\
\hline
\end{tabular}

\section{The 1993 Nobel Laureates}

R.A. Hulse is a Principal Research Physicist with the Tokamak Fusion Test Reactor facility at Princeton, New Jersey, USA. He studied at Cooper Union and went on to do graduate work at the University of Massachusetts, receiving his Ph.D. in 1975 for thesis research under Professor Taylor on pulsars that included the discovery in 1974 of the binary pulsar. He joined the National Radio Astronomy Observatory as a postdoc before moving to Princeton University's Plasma Physics Laboratory in 1975

J.H. Taylor has been the James S. McDonnell Distinguished University Professor of Physics at Princeton University since 1986. He studied at Haverford College and received his Ph.D. from Harvard University in 1968, where he worked as a Research Fellow for a year until joining the University of Massachussetts as an Assistant Professor, becoming Professor of Astronomy in 1977. He moved to Princeton in 1980. Professor Taylor's many honours and awards include the 1992 Wolf Prize in Physics.
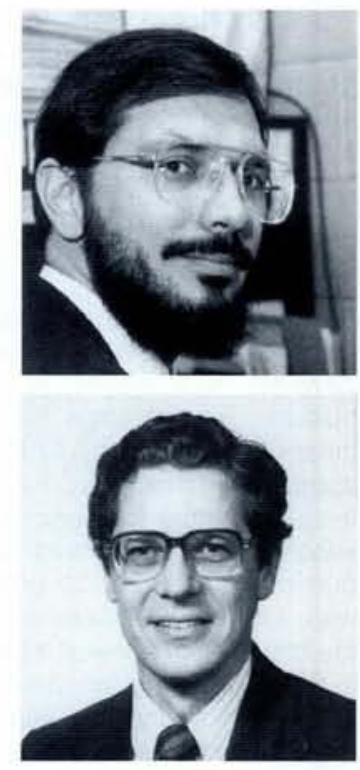

through a multi-channel spectrometer equipped with square-law detectors for each frequency channel. Digital signal averages accumulate a pulsar's average periodic waveform separately for each channel, using circuitry accurately synchronized with the observatory's time and frequency standard. A programmable synthesizer compensates for changing Doppler shifts caused by known accelerations of the pulsar and the observatory. Integrated profiles are recorded once every few minutes, together with appropriate time tags. The phase offset of an individual profile is measured relative to a high signal-to-noise standard integration, thereby yielding a topocentric time of arrival, or 'TOA', according to the observatory's master clock. TOAs obtained for different spectral channels are combined after correcting for dispersive delays caused by the ionized interstellar medium, and clock offsets measured by means of Earthorbiting GPS satellites [Lewandowski \& Thomas, 1991] are applied to correct all TOAs to the best available standard of terrestrial atomic time".

The phenomenological parameters appearing in the timing formula (Eq.1) are extracted from a set of TOAs by calculating the expected time of emission $\left\{T_{N}\right\}$ for the pulse numbers $\left\{N_{i}\right\}$ by combining Eq. 1 and Eq. 2 , and then minimizing the weighted sum of squared residuals with respect to all parameters to be determined. Details of the procedure are described in $[8,9]$.

\section{Confirmation of the Gravitational Radiation Damping}

Several thousand of TOAs for PSR $1913+16$ have been recorded at Arecibo since the discovery of the pulsar in the summer of 1974. These data determine the five Keplerian parameters and $\dot{\omega}$, the largest post-Keplerian parameter, to a few parts per million or better, as can be seen from Table 1 (note in particular the large value of $\dot{\omega}$ in comparison to the 43 " per century for Mercury). Two further post-Keplerian parameters, $\gamma$ and $\dot{P}_{\mathrm{b}}$, have now been determined with fractional accuracies better than
$0.3 \%$. According to what has been said earlier, this allows for a clean and precise test of general relativity.

A very interesting astrophysical application of general relativity should be mentioned at this point. The two post-Newtonian parameters $\dot{\omega}$ and $\gamma$ have different dependencies on the pulsar and companion masses, $m_{1}$ and $m_{2}$, and provide the most precise neutron star masses. It is highly significant for theories on late stellar evolution and neutron star formation in gravitational collapse that the masses determined in this manner are all close to the critical Chandrasekhar mass. For PSR 1913+16, the values (Fig. 2) are $m_{1}=1.4410(5) M_{c}$ and $m_{2}=1.3784(5) M_{0}$ while for the new binary pulsar system PSR B1534+12 discovered by Wolszczan [10], Taylor et al. [11] found very similar values: $m_{1}=1.34 \pm$ $0.07, m_{2}=1.34 \pm 0.07$.

We come now to the most important result of Taylor and collaborators. Knowing $\dot{\omega}$ and $\gamma$, general relativity predicts the rate of orbital period decay as a result of gravitational radiation of the binary pulsar system. In Fig. 3, this prediction is compared with the observed value of $\dot{P}_{\mathrm{b}}$. General relativity

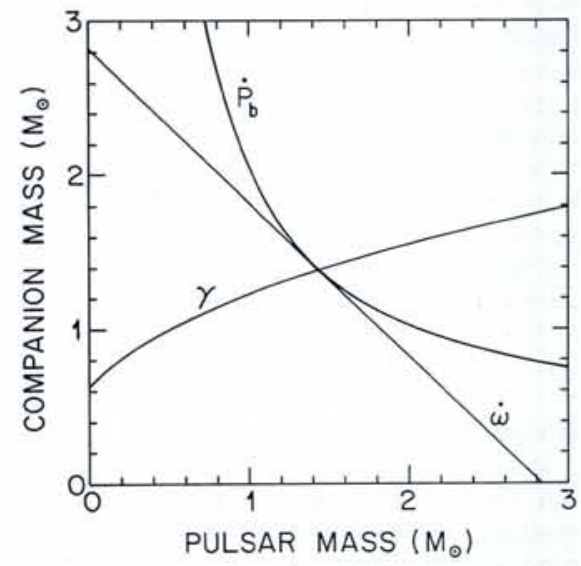

Fig. 2 - Curves in the planar mass-companion mass plane corresponding to the measured values of $\dot{\omega}, \gamma$ and $\dot{P}_{b}$ for PSR $1913+16$ (Table 1), if these are determined by general relativity. (Adapted from [3].) 


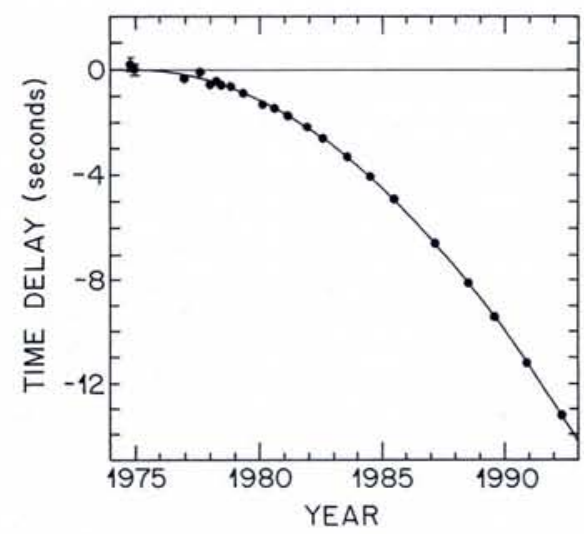

passes this " $\dot{\omega}-\gamma-\dot{P}_{b}$ " test perfectly at the present level of accuracy $(\approx 0.35 \%)$. It should be noted that the achieved accuracy is now so high that even a small correction for the effect of galactic acceleration needs to be included [12] (see Table 2 for details).

Within less than 10 years, two more binary pulsar systems, the one just mentioned (PSR B1534+12) and PSR B2127+11C, will presumably yield $\dot{\omega}-\gamma-\dot{P}_{\mathrm{b}}$ tests of gravitational radiation damping at the $1 \%$ level or
Fig. 3-Delay in the time of periastron of the binary pulsar PSR $1913+16$, relative to a non-dissipative model in which the orbital period remains fixed. Filled circles represent the measured shifts, while the smooth curve corresponds to the prediction of general relativity. (From [4].)

better [4]. In particular, PSR B1534+12 is a very interesting system because at least five post-Keplerian parameters can be accurately determined.

It should be clear from these brief remarks that the eight-hour binary pulsar PSR $1913+16$ has proven to be an outstanding laboratory for relativity. No relativist had ever dreamed of such a system before it was discovered. The high expectations held shortly after the discovery by Hulse and Taylor have been surpassed during the subsequent detailed study by Joe Taylor and his associates over almost 20 years. The work is an example of small science at its best and will survive as one of the most beautiful facets of physics in recent times. With it, general relativity has passed new, strong-field tests with complete success.
[1] Hulse R.A. \& Taylor J.H., Astrophys. J. 195 (1975) L51.

[2] Taylor J.H., Fowler A. and McCulloch P.M., Nature 277 (1979) 437; Taylor J.H. \& Weisberg J.M., Astrophys. J. 253 (1982) 908.

[3] ibid, 345 (1989) 434.

[4] Taylor J.H., Class. Quantum Gravity 1 (1993) 167.

[5] Blanford R. \& Teukolsky S.A., Astrophys. J. 205 (1976) 580; Epstein R., Astrophys. J. 216 (1977) 92 \& errata, 231 (1977) 644; Haugan M.P., Astrophys. J. 296 (1985) 1.

[6] Damour T. \& Deruelle N., Ann. Inst. H. Poincaré (Physique Théorique) 43 (1985) 107; ibid 44 (1986) 263.

[7] Damour T., 300 Years of Gravitation, Eds. S.W. Hawking \& W. Israel (Cambridge Univ. Press) 1982; idem., Proc. 2nd Canadian Conf. on General Relativity \& Relativistic Astrophysics, Eds.: A. Coley, C. Dyer \& T. Tupper (World Scientific Press) 1988.

[8] Damour T. \& Taylor J.H., Phys. Rev. D 45 (1992) 1840.

[9] Taylor J.H., Phil. Trans. R. Soc. A 341 (1992) 117.

[10] Wolszczan A., Nature 350 (1991) 688.

[11] Taylor J.H. et al., Nature 355 (1992) 132.

[12] Damour T. \& Taylor J.H., Astrophys. J. 366 (1991) 501.

\section{ACTIVE GALACTIC NUCLEI}

\section{Extreme UV Now the Issue}

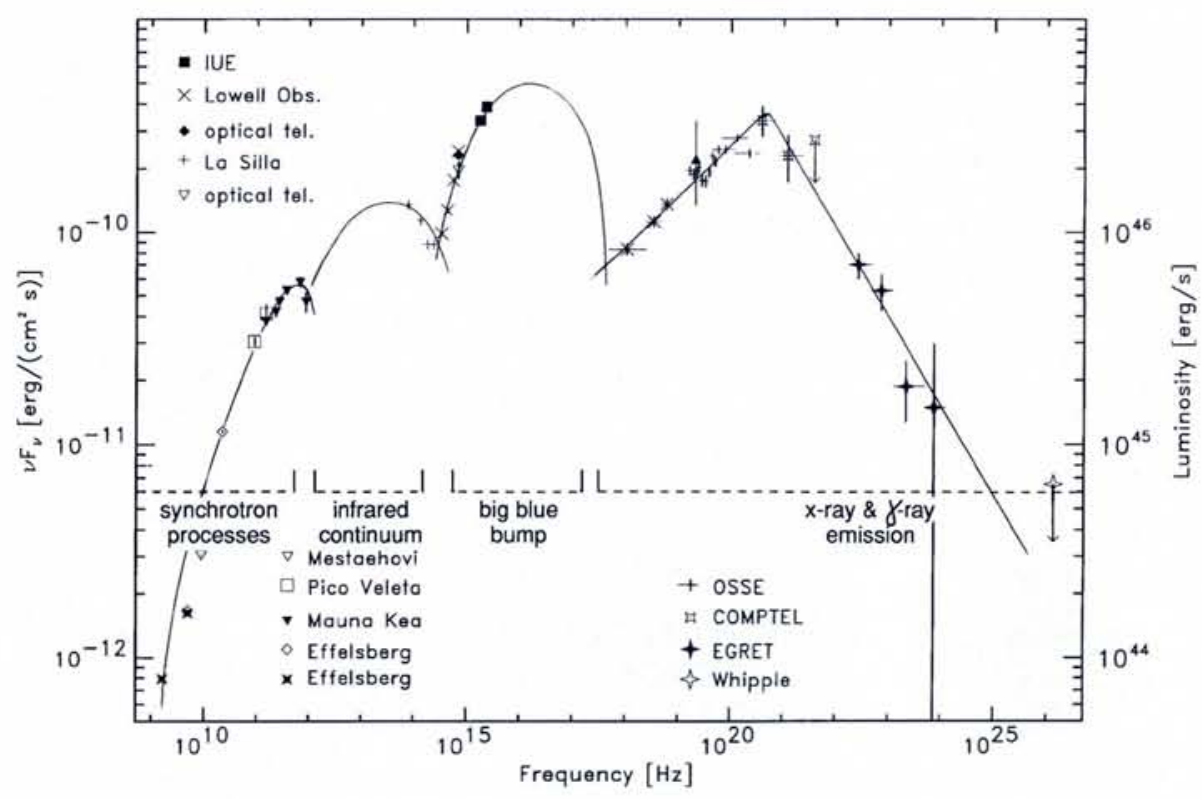

Active galactic nuclei (AGN) represent the brightest objects in the Universe. Energy emitted by AGN is distributed across the whole electromagnetic spectrum owing to different physical processes that are far from understood (Fig. 1). As variations of the energy flux are observed in all spectral bands on relatively short time-scales, the main goals of several worldwide observational programmes currently underway are to measure the multi-wavelength continua of these sources with Earth- and space-based observatories, to map the variations, and to uncover correlations between the different emission components.
The International Astronomical Union's (IAU) 159th symposium entitled Active Galactic Nuclei across the Electromagnetic Spectrum (August 30 - September 3, 1993) organized by Geneva Observatory reviewed recent progress. Attended by 300 participants from 31 countries with 200 posters and 50 oral contributions, there were reports on theory and observations on AGN involving data spanning 15 decades of the electromagnetic spectrum. Participants were reminded by $L$. Woltjer (the President of the European Astronomical Society) that the occasion marked the 30th anniversary of the discovery of quasars that lie at the cores of
Fig. 1 - Energy-density spectrum of the Seyfert Type 1 quasar 3C 273 from quasisimultaneous observations obtained with ground-based observatories and with the satellites IUE, Ginga and CGRO [Lichti et al., this symposium]. This hitherto unpublished energy spectrum represents the first time that simultaneous observations spanning such a large frequency range have been combined. It can be divided into four main components each originating from different physical processes.

extremely active galaxies, and the 50th anniversary of Seyfert's discovery of a particular class of active galaxies.

Concerning the main topic of the symposium, namely multi-wavelength continuum emission of $A G N$ and its variability, two main groups of $A G N$ are now recognized. Recalling that models of AGN generally involve massive black holes and central jets surrounded by accretion disks threaded by magnetic fields (see cover illustration), in the socalled blazar class, the featureless emission can be understood using models involving shocks in the jets. In Seyfert-type objects, including both radio-quiet and radio-loud sources, emission is dominated by several components (Fig. 1) which are either undetectable or not present in the former class. While the synchrotron origin of the radio emission and the contribution of reprocessing by dust to the infrared continua are now widely accepted, the origins of the optical to soft x-ray component and of the hard $x$-ray to $\gamma$-ray continuum remain controversial.

\section{Hard X-Ray to $\gamma$-Ray Range}

Our knowledge of the hard $\mathrm{x}$-ray and $\gamma$-ray emission of AGN has increased rapidly in the last five years with the results, reviewed during the meeting, of the Ginga, Granat and Compton GRO satellites and of the groundbased Whipple TeV-observatory.

In a fraction of the active nuclei, which are thought to be observed perpendicular to the accretion disc (i.e., along the jet), the $\gamma$-rays 